\title{
Dillenia species: a review of the traditional uses, active constituents and pharmacological properties from pre-clinical studies
}

\begin{abstract}
Context: Dillenia (Dilleniaceae) is a genus of about 100 species of flowering plants in tropical and subtropical trees of Southern Asia, Australasia, and the Indian Ocean Islands. Until now, only eight Dillenia species have been reported to be used traditionally in different countries for various medical purposes. Out of eight species, D. pentagyna (Roxb), D. indica (Linn.) and D. suffruticosa (Griffith Ex. Hook. F. \& Thomsom Martelli) have been reported to be used to treat cancerous growth. Objective: The present review explored and provided information on the therapeutic potential of Dillenia species. Methods: Comprehensive and relevant literature on the therapeutic potential of Dillenia species was gathered through electronic databases including Google Scholar, Scopus, PubMed, and books, without limiting the dates of publication. Results and conclusion: The review demonstrated that only a few Dillenia species have been proven scientifically for their therapeutic potential in pre-clinical studies, including D. pentagyna, D. indica, D. papuana (Martelli), D. meliosmifolia (Hook. F. Ex. Thomsom) and D. suffruticosa (Griffith Ex Hook. F. \& Thomson). A few species of Dillenia have undergone isolation and characterization of compounds with lupeol and betulinic acids having tremendous pharmacological potential. Dillenia species warrant further studies on their therapeutic potential, which may eventually lead to the development of new drug candidates for treatment of various diseases.
\end{abstract}

Keyword: Active constituent(s); Dilleniaceae; Ethnomedicinal; Pharmacological 